Elfriede Fritz beschreibt in ihrem Beitrag den sehr viel erfolgreicheren Umsetzungsprozess von GM und Gender Budgeting in der österreichischen Finanzverwaltung. Sie zeigt die verschiedenen Ebenen des Umsetzungsprozesses auf und legt dar, wie mit dem Instrument der wirkungsorientierten Gesetzesfolgenabschätzung auch im Steuerrecht eine Gleichstellungsperspektive verankert werden kann.

Der Beitrag von Edeltraud Lachmayer diskutiert für die Steuerpolitik relevante distributive und allokative Genderziele. Gemeint sind Ziele, die eine faktische Gleichstellung zwischen den Geschlechtern anstreben.

Alle Beiträge betonen, dass die Beachtung von Gleichstellungsaspekten in der Steuerpolitik adäquate Durchsetzungsmechanismen voraussetzt. Gisela Färber spricht explizit den Normenkontrollrat als unabhängige Institution an.

Elisabeth Klatzer erarbeitet die Rolle von zivilgesellschaftlichen Interventionen. Steuerpolitik gilt gemeinhin als sehr technisches und einem kleinen Kreis vorbehaltenes Thema. Am Beispiel von Österreich und Kanada zeigt sie, wie zivilgesellschaftliche
Interventionen gelingen können und betont die Bedeutung auch außergewöhnlicher Aktionen, da die Steuerpolitik für die Lebensbedingungen aller Bürger von enormer Wichtigkeit ist.

Maria Wersig identifiziert in ihrem Beitrag Hürden für die Umsetzung von Gleichstellungszielen in der bundesdeutschen Steuerpolitik.

\section{Fazit}

Der vorliegende Sammelband mit 15 Beiträgen knüpft an bereits bestehende Debatten zum Ehegattensplitting in Deutschland an, will aber vor allem neuen Perspektiven aufzeigen. Es soll hinter die Kulisse geschlechtsneutraler Formulierungen geschaut werden und der Blick auf die tatsächlichen Verteilungs- und Anreizwirkungen von Steuern gelenkt werden. Die Beiträge aus Österreich, das schon seit den Siebzigern eine Individualbesteuerung für Ehepaare vorsieht und wo ein sogenanntes „Gender Budgeting“ 2009 in die Verfassung eingefügt wurde, und Deutschland erlauben es, unterschiedliche Steuersysteme gegenüberzustellen und so landesspezifische Perspektiven zu hinterfragen.

\title{
Aktuelle Pressemitteilungen und Stellungnahmen
}

\section{Pressemitteilungen}

14-05 Frauenorganisationen fordern besseren gesetzlichen Gewaltschutz - One Billion Rising for Justice (13.2.2014)

14-04 Gremien für Bundesrichterwahlen signifikant weiblicher (4.2.2014)

14-03 djb lehnt „Rente mit 63“ ab und fordert Nachbesserungen bei der „Mütterrente“ (31.1.2014)

14-02 djb zum Koalitionsvertrag aus frauenpolitischer Sicht - was verspricht er, was wird er halten, wozu schweigt er? (15.1.2014)

14-01e Press Release: German Women Lawyers Association (djb) calls on Greek Presidency to advance equal opportunities initiatives (7.1.2014)

14-01 djb fordert griechische Ratspräsidentschaft auf, gleichstellungspolitische Initiativen voranzubringen (7.1.2014)

13-37 Gemeinsame PM: Spitzenfrauen gratulieren Schwesig und Nahles. Starke Frauen braucht das Land (15.12.2013)

13-36 djb gratuliert: Verdienstorden für Jutta Wagner, djbPräsidentin 2005-2011 (10.12.2013)

13-35 „Sag mir, wo die Frauen sind.“ Aktionärinnen fordern Gleichberechtigung - 2009 bis 2013. Mehr Frauen in Führungspositionen, Fazit und Forderungen (28.11.2013)

13-34 Gemeinsame PM: Die Zukunft ist weiblicher: Vertreterinnen und Vertreter aus Wirtschaft, Wissenschaft und Zivilgesellschaft sprechen sich gemeinsam für gleichberechtigte Karrierechancen für Frauen und Männer in deutschen Unternehmen aus (28.11.2013)

\section{Stellungnahmen}

14-01 Diskussionsbeitrag zum migrations- und flüchtlingspolitischen Teil der Post-Stockholm-Strategie (Januar 2014)

13-11 Diskussionsbeitrag zum kommenden Justizprogramm der Europäischen Union (Post-Stockholm-Strategie) (Dezember 2013)

\section{LAUfENDE INFORMATIONEN ÜBER DIE ARBEIT DES DJB}

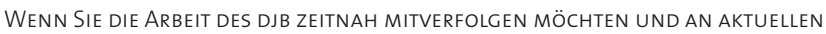
INFORMATIONEN INTERESSIERT SIND, KÖNNEN SIE SICH UNSERE PRESSEMITTEILUNGEN, UNSERE STELLUNGNAHMEN UND/ODER UNSERE VERANSTALTUNGSANKÜNDIGUNGEN PER E-MAIL ZUSCHICKEN LASSEN. AUF DER FOLGENDEN INTERNETSEITE KÖNNEN SIE SICH FÜR DIE ENTSPRECHENDEN NEWSLETTER REGISTRIEREN: HTTPS://WWW.DJB.DE/ST-PM/SERVICE/. AB SOFORT FINDEN SIE DEN DJB AUCH AUF FACEBOOK! 